\title{
A Remodelled Protease That Cleaves Phosphotyrosine Substrates
}

\author{
Zachary A. Knight $^{\dagger}$, Jennifer L. Garrison ${ }^{\dagger}$, Karina Chan ${ }^{\dagger}$, David S. King ${ }^{\ddagger}$, and Kevan M. \\ Shokat ${ }^{,} \dagger$ \\ tHoward Hughes Medical Institute, Department of Cellular and Molecular Pharmacology, University \\ of California, San Francisco, California 94107 \\ ‡Howard Hughes Medical Institute, Department of Chemistry, University of California, Berkeley, \\ California 94720
}

Proteases are the largest family of enzymes in the human genome and play central roles in a wide range of physiological processes. ${ }^{1}$ To carry out these diverse functions, proteolytic enzymes have evolved highly tuned substrate specificities that allow them to degrade specific cellular proteins in response to appropriate signals. For many proteases, this specificity is encoded by the amino acid sequence of the polypeptide substrate surrounding the bond that is cleaved (the scissile bond). For example, trypsin recognizes basic residues such as lysine or arginine, and a great diversity of such substrate preferences has been described. ${ }^{2,3}$ Remarkably, the substrate specificity of known proteases is limited to recognition of the 20 proteogenic (unmodified) amino acids: no protease has been shown to preferentially cleave its substrates adjacent to a site of post-translational modification, such as phosphorylation. Such enzymes would potentially find widespread application either as analytical tools for proteomics ${ }^{4}$ or as catalysts for the synthesis of novel biopolymers. ${ }^{5-7}$

Tyrosine phosphorylation is a dominant signaling mechanism in metazoans that links extracellular hormonal cues to the activation of intracellular signaling pathways. Herein we report efforts to design a protease that selectively cleaves its substrates adjacent to phosphotyrosine residues. Site-directed mutagenesis has been widely used to probe and modify the catalytic properties of enzymes, ${ }^{8-13}$ and the bacterial protease subtilisin BPN' was a model system for many early efforts in protein design. ${ }^{12-19}$ Subtilisin variants have been identified that display enhanced activity in organic solvents, ${ }^{20,21}$ stability at extremes of $\mathrm{pH}^{14,21}$ or temperature, ${ }^{15,21}$ and resistance to chemical oxidation. ${ }^{16}$ Subtilisin's catalytic properties have been altered to introduce peptide ligase $e^{5,6}$ or peroxidase ${ }^{22,23}$ activity, and its substrate specificity has been tuned to favor specific hydrophobic or electrostatic features. ${ }^{17-19}$ Many of these changes have been rationalized through structural analysis of subtilisin mutants, making this enzyme a well-characterized template for the design of novel proteolytic specificity.

Wild-type subtilisin BPN' exhibits a strong preference for peptide substrates containing phenylalanine or tyrosine at the residue directly N-terminal to the scissile bond (the P1 position). This specificity is controlled by residues that line a substrate binding pocket in the protease (the $\mathrm{S} 1$ pocket) that makes contact with the $\mathrm{P} 1$ aromatic side chain. We sought to

(c) 2007 American Chemical Society

shokat@cmp.ucsf.edu.

Supporting Information Available: Supplementary Table 1 and Figure 1 as well as complete experimental protocols. This material is available free of charge via the Internet at http://pubs.acs.org. 
remodel the S1 substrate binding pocket of subtilisin BPN' to selectively enhance its activity toward phosphotyrosine substrates.

A panel of peptide substrates were synthesized in which the P1 residue was varied as either phosphotyrosine, tyrosine, or phenylalanine. The residues surrounding P1 were fixed to an optimal subtilisin substrate sequence in order to direct peptide cleavage $\mathrm{N}$-terminal to the $\mathrm{P} 1$ residue. ${ }^{24}$ Finally, the aminobenzoic acid (Abz)-nitrotyrosine $\left(\mathrm{Y}^{\mathrm{NO} 2}\right)$ FRET donor-quencher pair was incorporated so that peptide cleavage could be monitored by fluorescence. ${ }^{24}$ Using these substrates, wild-type subtilisin BPN' cleaved a phosphotyrosine peptide approximately 500 -fold less efficiently $\left(k_{\text {cat }} / K_{\mathrm{M}}\right)$ than the matched tyrosine or phenylalanine substrates (Figure 1).

Modeling of a phosphotyrosine side chain into the $\mathrm{P} 1$ pocket of subtilisin suggested multiple residues that could potentially make contacts with the tyrosine phosphate. We therefore systematically mutated the residues lining the $S 1$ pocket of subtilisin to introduce basic residues or hydrogen bond donors that might make favorable interactions with the negatively charged phosphotyrosine moiety. These 18 single mutants were expressed, purified, and assayed against model peptide substrates.

Mutations at several positions in the S1 pocket enhanced selectivity for phosphotyrosine substrates. Mutation of Glu156 to the basic residues arginine or lysine had a dramatic effect on substrate selectivity, resulting in 200 -fold enhancement in selectivity for phosphotyrosine relative to tyrosine or phenylalanine (Figure 1). This improvement was achieved primarily through a reduction in the $K_{\mathrm{M}}$ for phosphotyrosine peptides (Supporting Information Table 1). Glu156 sits in a solvent-exposed region at the entrance to the S1 binding cleft, and modeling suggests that the side chain of Glu156 would be in close proximity to the phosphate moiety of bound phosphotyrosine substrates (Supporting Information Figure 1).

To search for variants with further selectivity for phosphotyrosine, Glu156 mutations were combined with second site mutations to generate a panel of 21 double mutants that were expressed, purified, and assayed (Figure 1). Several of these double mutants showed greater than 10-fold additional enhancements in phosphotyrosine selectivity, resulting in enzymes that preferentially cleave phosphotyrosine relative to the optimal wild-type substrate. The most selective mutants combine an E156R or E156K mutation with the substitution P129G. Pro129 sits at the entrance to the $\mathrm{S} 1$ binding cleft directly opposite Glu156, where the cavity created by the P129G mutation could potentially accommodate the phosphate moiety (Supporting Information Figure 1). The double mutant E156R/P129G cleaved a phosphotyrosine substrate 5 -fold more efficiently than the optimal wild-type substrate, representing a greater than 2500fold switch in substrate selectivity relative to the wild-type enzyme. The double mutant enzyme is expressed at levels comparable to the wild-type and has high specific activity $\left(k_{\mathrm{cat}}=37.1\right.$ $\mathrm{s}^{-1}$ ), suggesting that the selectivity improvement for phosphotyrosine is achieved without impairing the stability or catalytic efficiency of the enzyme.

We next sought to characterize how these mutations influence the selectivity of the double mutant for alternative P1 substrates. Optimal peptide substrates were synthesized containing sulfotyrosine (sTyr), phosphoserine (pSer), and phosphothreonine (pThr) amino acids at the P1 position, and the E156R/P129G enzyme was assayed against these substrates (Table 1). Sulfotyrosine was utilized only 2-fold less efficiently than phosphotyrosine, consistent with the fact that these amino acids are isosteric and negatively charged at neutral $\mathrm{pH}$. By contrast, phosphothreonine and phosphoserine peptides were extremely poor substrates for the modified enzyme, with catalytic efficiencies diminished approximately 36000 -fold and 76000 -fold, respectively, relative to the matched phosphotyrosine peptide. The narrow S1 binding of cleft of subtilisin makes close hydrophobic interactions with the aromatic P1 side chain of the 
substrate that sterically preclude the binding of $\beta$-branched amino acids at this site; the selectivity we observe relative to phosphoserine and phosphothreonine suggests that these interactions have been preserved in the double mutant.

We further characterized the selectivity of E156R/P129G by assaying its activity against a commercial panel of protease substrates that vary in the identity of the P1 amino acid (Table 1). Within this panel, phenylalanine was the optimal residue at P1, suggesting that the selectivity of E156R/P129G for non-phosphorylated substrates was not significantly altered relative to the wild-type enzyme. Importantly, aspartate and glutamate remained very poor P1 substrates for the modified enzyme (utilized 670 and 245 -fold less efficiently relative to phenylalanine), indicating that the mutations which enhance phosphotyrosine binding do not nonspecifically bias the enzyme toward all acidic substrates.

The identity of the P1 residue is a critical determinant of substrate binding to subtilisin BPN'. However, amino acids at adjacent positions contribute to substrate recognition, ${ }^{24}$ and it will be important to explore how these preferences affect the cleavage patterns produced when complex mixtures of proteins are used as substrates. Alternatively, as the selectivity of adjacent subsites has been modified through protein engineering, ${ }^{25,26}$ it may be possible to introduce additional mutations that confer recognition of specific phosphotyrosine consensus sequences.

\section{Supplementary Material}

Refer to Web version on PubMed Central for supplementary material.

\section{Acknowledgments}

This work was supported by NIH RO1EB001987.

\section{References}

1. Marnett AB, Craik CS. Trends Biotechnol 2005;23:59-64. [PubMed: 15661339]

2. Harris JL, Backes BJ, Leonetti F, Mahrus S, Ellman JA, Craik CS. Proc Natl Acad Sci USA 2000;97:7754-9. [PubMed: 10869434]

3. Gosalia DN, Salisbury CM, Ellman JA, Diamond SL. Mol Cell Proteomics 2005;4:626-36. [PubMed: 15705970]

4. Knight ZA, Schilling B, Row RH, Kenski DM, Gibson BW, Shokat KM. Nat Biotechnol 2003;21:1047-54. [PubMed: 12923550]

5. Abrahmsen L, Tom J, Burnier J, Butcher KA, Kossiakoff A, Wells JA. Biochemistry 1991;30:41519. [PubMed: 2021606]

6. Nakatsuka T, Sasaki T, Kaiser ET. J Am Chem Soc 1987;109:3808-10.

7. Jackson DY, Burnier J, Quan C, Stanley M, Tom J, Wells JA. Science 1994;266:243-7. [PubMed: 7939659]

8. Ghosh SS, Bock SC, Rokita SE, Kaiser ET. Science 1986;231:145-8. [PubMed: 3510454]

9. Gardell SJ, Craik CS, Hilvert D, Urdea MS, Rutter WJ. Nature 1985;317:551-5. [PubMed: 3840231]

10. Craik CS, Roczniak S, Largman C, Rutter WJ. Science 1987;237:909-13. [PubMed: 3303334]

11. Craik CS, Largman C, Fletcher T, Roczniak S, Barr PJ, Fletterick R, Rutter WJ. Science 1985;228:291-7. [PubMed: 3838593]

12. Carter P, Wells JA. Science 1987;237:394-9. [PubMed: 3299704]

13. Carter P, Wells JA. Nature 1988;332:564-8. [PubMed: 3282170]

14. Cunningham BC, Wells JA. Protein Eng 1987;1:319-25. [PubMed: 3334089]

15. Pantoliano MW, Ladner RC, Bryan PN, Rollence ML, Wood JF, Poulos TL. Biochemistry 1987;26:2077-82. [PubMed: 3476160]

16. Estell DA, Graycar TP, Wells JA. J Biol Chem 1985;260:6518-21. [PubMed: 3922976] 
17. Russell AJ, Fersht AR. Nature 1987;328:496-500. [PubMed: 3302724]

18. Wells JA, Cunningham BC, Graycar TP, Estell DA. Proc Natl Acad Sci USA 1987;84:5167-71. [PubMed: 3299378]

19. Wells JA, Powers DB, Bott RR, Graycar TP, Estell DA. Proc Natl Acad Sci USA 1987;84:1219-23. [PubMed: 3547407]

20. Chen K, Arnold FH. Proc Natl Acad Sci USA 1993;90:5618-22. [PubMed: 8516309]

21. Ness JE, Welch M, Giver L, Bueno M, Cherry JR, Borchert TV, Stemmer WP, Minshull J. Nat Biotechnol 1999;17:893-6. [PubMed: 10471932]

22. Wu ZP, Hilvert D. J Am Chem Soc 1990;112:5647-5648.

23. Bell IM, Fisher ML, Wu ZP, Hilvert D. Biochemistry 1993;32:3754-62. [PubMed: 8385489]

24. Gron H, Meldal M, Breddam K. Biochemistry 1992;31:6011-8. [PubMed: 1627543]

25. Ballinger MD, Tom J, Wells JA. Biochemistry 1995;34:13312-9. [PubMed: 7577915]

26. Ballinger MD, Tom J, Wells JA. Biochemistry 1996;35:13579-85. [PubMed: 8885837] 
A.

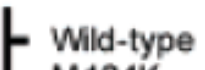

- M124K

- G127S

- A151S

- G154S

- E156G

- $\mathrm{E} 156 \mathrm{H}$

- E156N

- E156Q

- E156R

- E156K

- $\mathrm{G} 166 \mathrm{H}$

- G166N

- G1660

- G166S

- G166K

- G169R

- V174R $\log \left(\frac{k_{\text {cat }} / K_{\mathrm{M} \text { pTyr }}}{k_{\text {cat }} / K_{M} \text { Tyr } / \text { Phe }}\right)$

B.

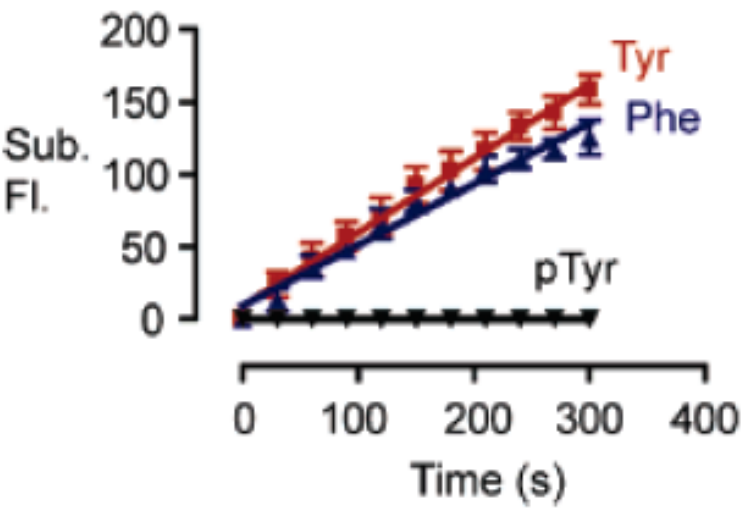

\section{Double mutants}

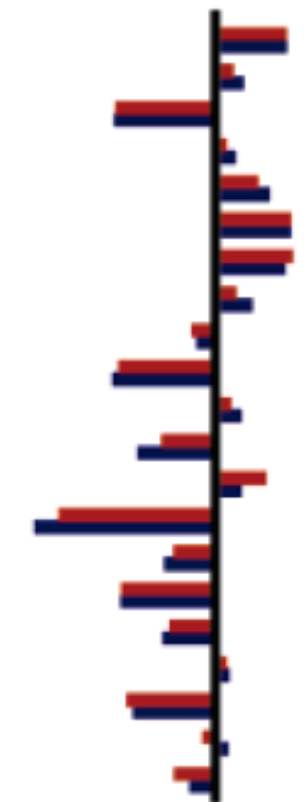

- E156R/G127S

- E156R/S191K

- E156R/S191R

- E156R/P129R

- E156R/P129K

- E156R/P129G

- E156K/P129G

- E156K/P129R

- E156K/P129K

- E156K/S191R

- E156K/S191K

- E156KN $174 \mathrm{~K}$

- E156KJG166K

- E156K/L135K

- E156K/Y104K

- E1560/M124K

- E156H/G166K

- E156G/G166K

- E156G/P129R

- E156G/S191K

- E156G/S191R

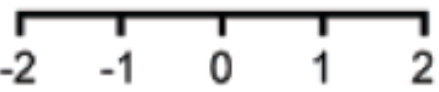

$\log \left(\frac{k_{\mathrm{cat}} / K_{\mathrm{M} \text { pTyr }}}{k_{\mathrm{cat}} / K_{\mathrm{M}} \text { Tyr/Phe }}\right)$

\section{E156R/P129G}

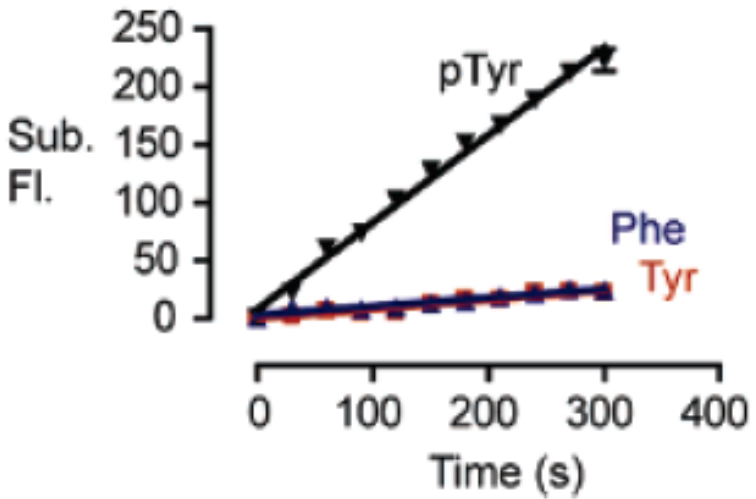

Figure 1.

(A) Plot of the log ratio of catalytic efficiencies for subtilisin single and double mutants assayed with the peptide substrates Abz-FRPXGFY ${ }^{\mathrm{NO} 2}$-D, where $\mathrm{X}$ is either Tyr, Phe, or pTyr. (B) Cleavage of the Abz-FRPXGFY ${ }^{\mathrm{NO}}$-D peptide $(0.8 \mu \mathrm{M})$ as a function of time by wild-type and E156R/P129G subtilisin. $Y$ axis: substrate fluorescence. 


\section{Table 1}

Catalytic Efficiency of E156R/P129G Subtilisin with Peptide Substrates

\begin{tabular}{lcccc}
\hline \multicolumn{2}{c}{ Abz-FRPXGFY ${ }^{\text {NO2 }}$-D } & & \multicolumn{2}{c}{ Suc-AAPX-pNA } \\
\cline { 1 - 1 } $\mathbf{P 1}$ & $\boldsymbol{k}_{\text {cat }} / \boldsymbol{K}_{\mathbf{M}}\left(\mathbf{M}^{-\mathbf{1}} \mathbf{s}^{-\mathbf{1}}\right)$ & & P1 & $\boldsymbol{k}_{\text {cat }} / \boldsymbol{K}_{\mathbf{M}}\left(\mathbf{M}^{-\mathbf{1}} \mathbf{s}^{-\mathbf{1}}\right)$ \\
\hline pTyr & $1.41 \times 10^{7}$ & & Phe & $8.64 \times 10^{4}$ \\
sTyr & $7.27 \times 10^{6}$ & Leu & $2.26 \times 10^{3}$ \\
Tyr & $2.71 \times 10^{6}$ & Ala & $5.93 \times 10^{2}$ \\
Phe & $2.71 \times 10^{6}$ & Val & $1.89 \times 10^{1}$ \\
pSer & $3.90 \times 10^{2}$ & Ile & $2.33 \times 10^{1}$ \\
pThr & $1.85 \times 10^{1}$ & Asp & $1.29 \times 10^{2}$ \\
& & Glu & $3.53 \times 10^{2}$ \\
& & Arg & $1.30 \times 10^{1}$ \\
& & Lys & $5.28 \times 10^{1}$ \\
\hline
\end{tabular}

